\title{
Training and Learning Curve in Chronic Cerebrospinal Venous Insufficiency Assessment
}

read with interest the article entitled "Mystery of Chronic Cerebrospinal Venous Insufficiency: Identical Venographic and Ultrasound Findings in Patients with MS and Controls" by McAuliffe and Kermode ${ }^{1}$ published on-line in January by the American Journal of Neuroradiology. I can, therefore, disagree with both the methodology and conclusions of the authors.

It is not clear whether the duplex operators had sufficient training for searching eventual jugular vein abnormalities and actually did the search following the usual required protocol. The authors did not cite the protocol guidelines published by 7 international scientific societies. ${ }^{2}$ In addition, it has been proved that training is mandatory to improve reproducibility of chronic cerebrospinal venous insufficiency (CCSVI) screening. The $\kappa$ coefficient found was significantly different when comparing interobserver variation in trained-versus-untrained sonographic operators. ${ }^{3}$

Thirty cases are not enough to reach statistically significant results. In addition, it has been proved that training is mandatory to improve reproducibility of CCSVI screening. This methodology is very different from that of Zamboni et al in their seminal article. ${ }^{4}$ CCSVI was proposed with a multimodality approach combining sonography abnormalities with imaging performed by catheter venography.

After 30 years' experience as a vascular surgeon and diagnostician, ${ }^{5}$ I can comment on the work of the authors because after
$>1500$ examinations for CCSVI research, I have matched $90 \%$ of abnormalities to patients with MS. Half of these were post-percutaneous venous angioplasty controls, all of whom submitted to venographic examinations that confirmed CCSVI.

Conclusions are made with large numbers and require time, proper training, and a learning curve, as well as comparison of Doppler sonography with the criterion standard catheter venography.

\section{REFERENCES}

1. McAuliffe W, Kermode AG. Mystery of chronic cerebrospinal venous insufficiency: identical venographic and ultrasound findings in patients with MS and controls. AJNR Am J Neuroradiol January 312013 [Epub ahead of print]

2. Zamboni P, Morovic S, Menegatti E, et al. Screening for chronic cerebrospinal venous insufficiency (CCSVI) using ultrasound: recommendations for a protocol. Int Angiol 2011;30:571-97. Erratum in: Int Angiol 2012;31:201

3. Menegatti E, Genova V, Tessari M, et al. The reproducibility of colour Doppler in chronic cerebrospinal venous insufficiency associated with multiple sclerosis. Int Angiol 2010;29:121-26

4. Zamboni P, Galeotti R, Menegatti E, et al. Chronic cerebrospinal venous insufficiency in patients with multiple sclerosis. J Neurol Neurosurg Psychiatry 2009;80:392-99

5. Bavera PM, Agus GB, Alpini D, et al. Results from 823 consecutive duplex exams for CCSVI in a vascular centre. Acta Phlebologica 2012;13:141-48

P.M. Bavera

Vascular Surgery University of Milan Milan, Italy 